\title{
Bio-Inspired Algorithms for Dynamic Resource Allocation in Cognitive Wireless Networks
}

\author{
T. Renk, C. Kloeck, D. Burgkhardt, and F. K. Jondral \\ Institut für Nachrichtentechnik \\ Universität Karlsruhe (TH), Germany \\ $\{$ renk, kloeck, burgkhardt, fj $\} @$ int.uni-karlsruhe.de
}

\author{
D. Grandblaise, S. Gault, and J. C. Dunat \\ Motorola Labs Paris \\ Parc-les-Algorithmes, France \\ \{david.grandblaise, sophie.gault, jcdunat\}@motorola.com
}

\begin{abstract}
Regulation will experience enormous changes in the near future resulting in seamless connectivity by bluring spectrum borders. A promising approach in this context is dynamic spectrum allocation which leads to a more flexible access to spectral resources by employing intelligent radio devices called cognitive radios. This paper is concerned with bio-inspired approaches that exploit distribution in multi-radio environments where many users have to share a finite resource harmoniously. Three applications of bio-inspired techniques are described. The first one deals with the detection of spectrum holes and the second one describes resource allocation in orthogonal frequency division multiple access based systems. The third one is concerned with distributed resource auctioning.
\end{abstract}

\section{INTRODUCTION}

In the near future wireless communications will bear little similarities to currently used systems. The demand for multimedia communications and seamless access to heterogeneous mobile networks is extremely rising and this tendency is expected to continue in the next few years. Unfortunately, spectrum allocation today is very inflexible. A further drawback due to complicated and time-consuming regulatory issues is an inefficient utilization of spectral resources. In order to overcome these barriers, advanced resource access algorithms and sharing techniques must be employed [1], [2]. Here, resource is not only restricted to the most common terms of power and bandwidth, but also means time-slots in TDMA systems, (orthogonal) codes in CDMA systems, and different sub-carriers in OFDMA systems. Furthermore, all thinkable combinations of the above mentioned aspects can also be considered as resources.

Sharing processes can be seen as interaction between different individuals in a social community. This step creates the ability that a group of identical individuals (cognitive radios in this purpose) executes complex tasks which would not be possible if each cognitive radio acted as a standalone device. Consequently, collaboration among several radios within multi-user environments must be exploited. In addition, suitable sharing algorithms can only be employed if individual quality of service (QoS) constraints for multimedia applications are met in an efficient and accomplishable manner.

After highlighting the advantages of swarm intelligence, the detection of spectrum holes and resource allocation in OFDMA based systems are described in Section II. The next section deals with distributed resource auctioning, where a specific belief function is introduced that is an estimation of the cumulative distribution function. Finally, Section IV concludes the paper.

\section{SWARM INTELLIGENCE}

\section{A. Advantages of swarm intelligence}

Swarm intelligence based systems are very flexible and robust with respect to environmental constraints and disturbances which makes them very attractive for technical realizations [3], [4]. Moreover, swarm intelligence inherits some important advantages such as:

- Scalability: The number of individuals can be adapted to the network size.

- Fault tolerance: Since the behavior of a swarm is not controlled by a centralized entity, the loss of a few individuals does not cause catastrophic failure.

- Adaptation: The swarm can react to environmental changes due to the fact that each individual has the ability to adapt.

- Speed: Changes in the network can be spread very quickly.

- Modularity: Individuals act independently of other network layers.

- Autonomy: Little or no human control is required.

- Parallelism: Operations of individuals are executed in a parallel manner.

The following subsections describe two examples where swarm intelligence can applicatively be used.

\section{B. Detection of spectrum holes}

The detection of spectrum opportunities is one major task in the research area of dynamic spectrum allocation. This fact becomes even more important in the context of cognitive radio which is aware of environmental changes and has the ability to act accordingly. A well-known method for the detection of occupied spectrum is energy detection where a decision on use/no use of spectrum is simply done by judging the strength of the detected signal within a predefined bandwidth. Another possibility is cyclostationary based detection. Here, inherent characteristics of modulated signals are exploited that can additionally be used in the receiver for parameter estimation [5]. 


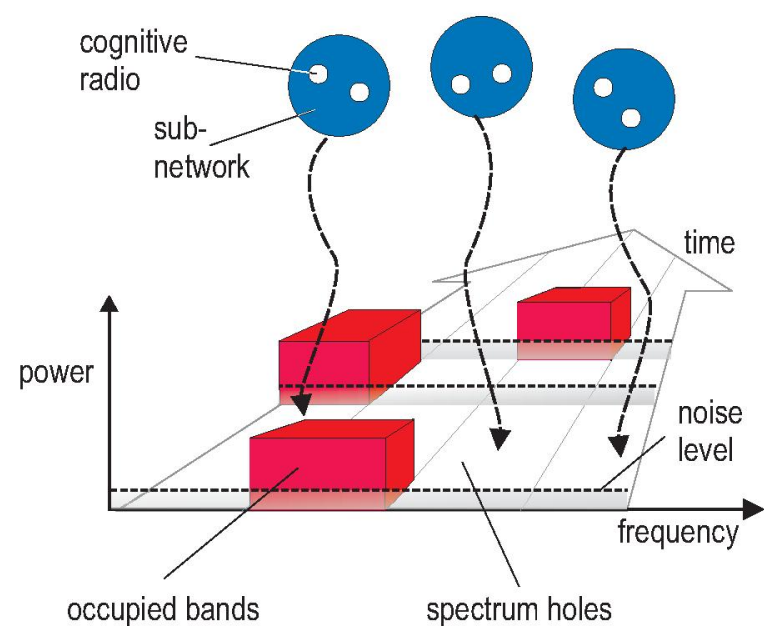

Fig, 1. Schematic description of the process of spectrum holes detection with respect to the three dimensions of time, frequency, and power. A channel is considered to be allocated if the power level exceeds the noise level. Here, $K=8, N=4$, and $M=2$.

This section describes the detection of spectrum holes in order to be able to build up reliable communications by using swarm intelligence based systems. The motivation for this approach comes from fauna, i.e., foraging, where every bird sees through the eyes of all the other birds, thus increasing the detection probability of finding food. It is true that each mobile terminal may scan the whole frequency range, but this obviously is no proper solution as it takes too much time and is too power-consuming [5].

Consider a network consisting of $K$ cognitive radios. These radios can then be merged into a swarm of $K$ individuals. In order to meet scalability issues, this swarm will then be divided into $N$ cognitive sub-networks where each sub-network consists of $M<K$ cognitive radios. With respect to interference issues, it becomes obvious that time, frequency, location, and QoS guarantees (this becomes more and more important due to an increasing demand for multi-media applications) must be considered for clustering. Clever clustering techniques help to reduce the search period enormously, whereas bad clustering not only enlarges the search period but can also lead to a higher degree of interference. For the sake of simplicity, each cognitive sub-network is meant to have the same number of cognitive radios (see Fig. 1). The cognitive sub-networks will then be scattered equidistantly over the frequency space. Let the overall bandwidth be denoted as $B$. Then, each cognitive sub-network is in charge of a bandwidth $B_{i}=B / N$, $i \in\{1,2, \ldots, N\}$. Eventually, each sub-network will be split up and each cognitive radio will scan a frequency range

$$
B_{i j}=\frac{B_{i}}{M}=\frac{B}{N \cdot M}, \quad j \in\{1,2, \ldots, M\},
$$

which is much smaller than it would be the case if a single mobile terminal would have to scan the whole range. Say the time it takes a single radio to scan the whole bandwidth $B$ is $\tau$, then this parallelizing process reduces scanning time for

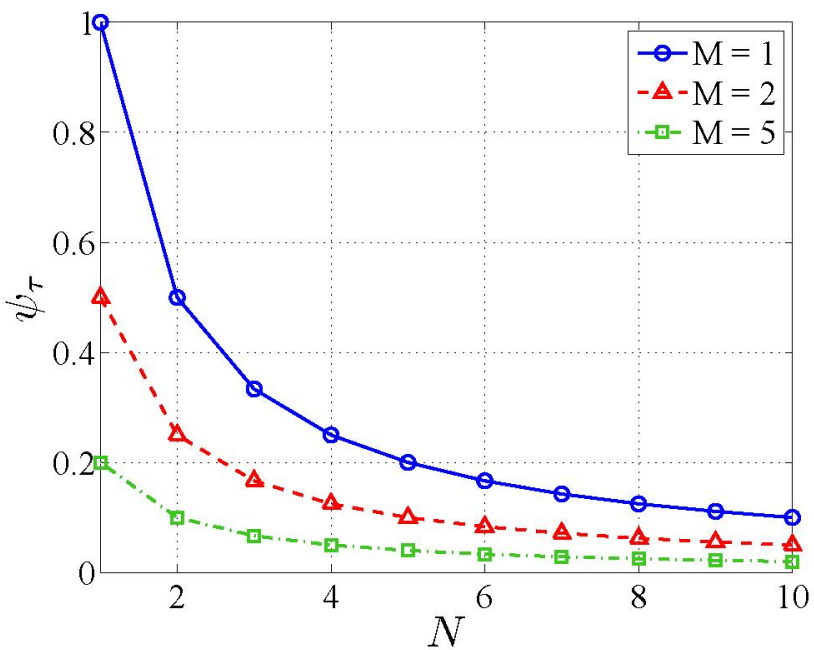

Fig. 2. Time saving facor, $\psi_{\tau}$, versus the number of cognitive sub-networks. Parameter is the number of cognitive radios within a sub-network.

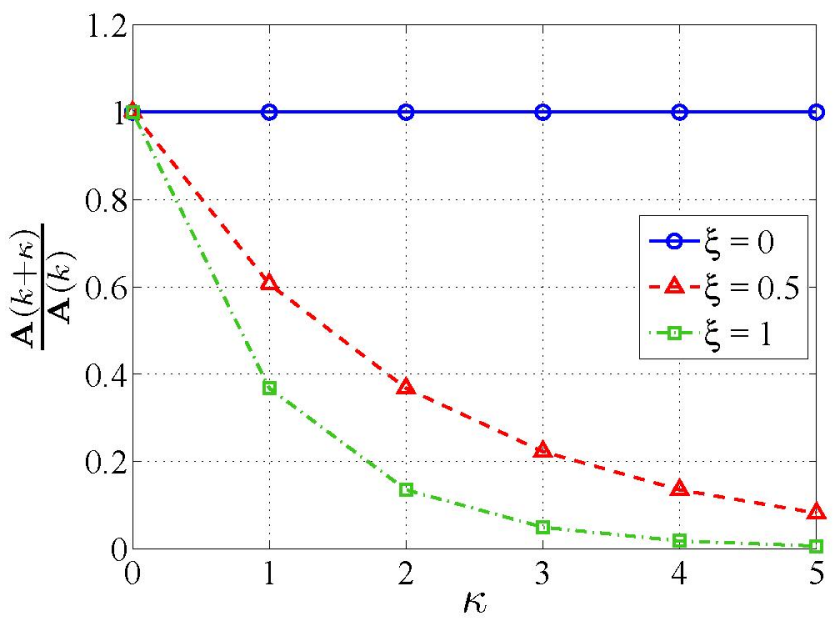

Fig. 3. Decay factor versus decay period $\kappa$. Parameter is the decay rate $\xi$.

each mobile terminal to

$$
\tau_{i j}=\frac{1}{N \cdot M} \cdot \tau=\psi_{\tau} \cdot \tau
$$

where $\psi_{\tau}$ is indicated as time saving factor. This factor is depicted versus the number of cognitive sub-networks, $N$, in Fig. 2. As parameter the number of cognitive radios in each sub-network, $M$, was used. For the simple scenario considered in this paper, it becomes clear that $\psi_{\tau}$ is the reciprocal of the number of cognitive radios in the swarm. Furthermore, it can be stated that $\left.\psi_{\tau}\right|_{N=1}=1 / M$ and $\lim _{N \rightarrow \infty}=0$. Of course, these simple relations do not hold any longer if information bearing beacon channels are previously excluded from the overall bandwidth and experience values in the shape of statistical expressions are included in the considerations.

After the scanning process each cognitive radio communicates its results to all other radios within its cognitive subnetwork via a common control channel. The control channel 
TABLE I

Numerical VAlues For the First SIMUlation PARAMEters (REFERS to Fig, 4 ANd Fig, 5).

\begin{tabular}{l|l|l} 
Notation & Description & Values \\
\hline \hline$\beta$ & Parameters controlling $f(x)$ function's shape & 0.4 \\
\hline$\epsilon$ & Parameter used to find out $x_{l}$ such that $f\left(x_{l}\right)=\varphi-\epsilon$ and $x_{u}$ such that $f\left(x_{u}\right)=-\varphi+\epsilon$ & $10^{-3}$ \\
\hline$\Theta_{\text {init }}$ & Initial threshold value after reset & 0 \\
\hline$\Theta_{\min }$ & Minimum possible threshold value $\Theta_{i j}$ & -50 \\
\hline$\Theta_{\max }$ & Maximum possible threshold value $\Theta_{i j}$ & +50 \\
\hline$n_{\min }$ & Minimum simultaneous number of sub-carriers used per active node & 1 \\
\hline$n_{\max }$ & Maximum simultaneous number of sub-carriers used per active node & 1 \\
\hline$M$ & Number of nodes & {$[10: 10: 100]$} \\
\hline$N$ & Number of available sub-carriers to allocate & 20 \\
\hline
\end{tabular}

can either be

1) a dedicated frequency channel beyond the utilized frequencies or

2) a channel that is expanded over the whole utilized frequency range by applying code spreading techniques.

For the broadcasting of the information each cognitive radio has to write its results into its assigned row of the $M \times L$ allocation matrix $\mathbf{A}(\cdot)$. The number of columns, $L$, depends on the frequency resolution. Since most real-world problems change with time, the dynamic behavior of spectrum occupation must be taken into account. Therefore, a decay factor is introduced whose purpose can be compared to that of pheromones in ant colonies [3]. Pheromones enable ants to find the shortest path towards food and back. Each ant lays a trail of pheromone while walking towards the food and each ant prefers the path with the highest amount of pheromone. Because of the decay factor, allocation information decreases exponentially with time. This yields to

$$
\mathbf{A}(k+\kappa)=\mathbf{A}(k) \cdot \exp (-\xi), \quad \xi \geq 0,
$$

where $k$ describes the time variable, $\kappa$ is the decay period, $\exp (-\xi)$ is the above mentioned decay factor, and $\xi$ is the decay rate. Though the decay rate might be changed with time, it remains constant over one decay period thus leading to a linear decrease. Fig. 3 illustrates the decay factor versus the decay rate $\kappa$ for three different parameter values of $\xi$. It can easily be seen that

$$
\lim _{\kappa \rightarrow \infty} \mathbf{A}(k+\kappa)=\left\{\begin{array}{rll}
\mathbf{A}(k) & : & \xi=0 \\
0 & : & \xi>0
\end{array} .\right.
$$

After each cognitive radio in a sub-network is satisfied, information on left spectrum opportunities can be communicated between the masters of each sub-network. The master might be the first cognitive radio that claims this title via broadcasting a beacon. With this information it is possible for cognitive radios of other sub-networks that momentarily suffer from spectrum scarcity to connect properly. Another possibility after convergence is a principle that is similar to the anticonvergence principle introduced in [6]. All radios that did not have the chance to find proper spectrum opportunities to build up communications form a new swarm and the whole process starts again. This sounds logical since spectrum allocation is a highly dynamic process that changes in time.

\section{Resource allocation in OFDMA based systems}

1) Background: One important challenge in the management of a population of cognitive radios is how the available radio resource can be assigned in an optimal way to each individual cognitive radio without a central controller [7]. With respect to that question, this paragraph addresses how a distributed allocation technique inspired from swarm intelligence can allocate uplink sub-carriers between cognitive radios using OFDMA as a multiple access method in a single cell. Purpose is to derive a distributed scheme which enables on one hand the optimization of the uplink sum channel capacity in that cell while minimizing the signalling overhead on the other. In the followed approach, related to the propagation conditions experienced on each sub-carrier, each cognitive radio can individually assess its own perceived channel capacity for each available sub-carrier. An efficient distributed allocation technique can be achieved if each cognitive radio can communicate its local knowledge about its own assessment of each sub-carrier channel capacity to the other cognitive radios of the cell. Based on a WLAN like system, each cognitive radio can report this information to the access point. Then, the access point can broadcast the minimum and the maximum channel capacity experienced by all the users. Based on this information, each cognitive radio can individually run an iterative algorithm [8] to make its own decision about the sub-carriers it has to be assigned with. With this approach, although information sharing between cognitive radios is enabled with a central entity (i.e., access point), the final decision-making on the choice of the sub-carriers allocation for each cognitive radio is made individually by each cognitive radio. The implementation of such an approach requires the design of an appropriate medium access control structure to convey the shared information and to support the iterative negotiation process between the dispersed cognitive radios in the cell as well.

2) Learning and forgetting capabilities: This part examines how far control parameters for learning and forgetting capabilities can have an impact on the performance of the radio resource allocation algorithm [8]. The following results show how the system's learning and forgetting capabilities during the negotiation phase can be controlled.

The system's memory (composed of all the users) during a negotiation phase is controlled by the following parameters 


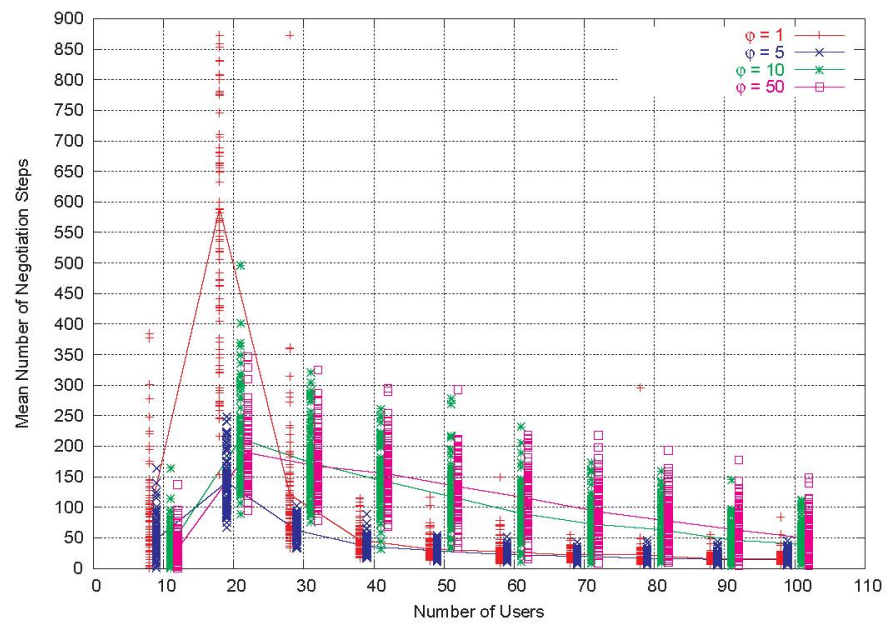

Fig. 4. Number of negotiation steps as a function of $\varphi$.

that require to be optimized together: $\varphi, \Theta_{\min }$, and $\Theta_{\max }$. The scenario under consideration is depicted in Table I.

Fig. 4 shows the mean and live values for the number of negotiation steps as a function of $\varphi$. Note that for readability purposes, the results for each value of number of users has a small offset in the horizontal axis. The standard deviation reduces as the number of users increases. Best results (low number of negotiation steps and low dispersion of values) are obtained for $\varphi=5$. Moreover, Fig. 4 shows that properly tuning of the system's learning and forgetting capabilities has an important impact on the system's negotiation duration. Especially when considering the system's negotiation duration, the difference between $\varphi$ and the $\Theta$ extreme limits $\left(\Theta_{\min }\right.$ and $\Theta_{\max }$ ) should not be too small (users become instable deciders with almost no memory of past experiences) and not be too important (users become low learners). $\varphi$ constraints the maximum $\Delta \Theta_{i j}$ achievable value that is updated after each negotiation time slot which feeds $\Theta_{i j}$.

Fig. 5 shows the mean and live values for the total sum capacity as a function of $\varphi$. Note that for readability purposes, the results of each value of the users have a small offset in the horizontal axis, too. The standard deviation reduces as the number of users increases. Furthermore, Fig. 5 shows that, as far as the total sum capacity is concerned, there is almost no impact of the memory (no major difference in the standard deviation of values as $M$, i.e., the number of users, increases).

\section{Distributed RESOURCE AUCTIONING}

Current communication systems bill the customer for providing services in cells where the capacity is not utilized completely by demanding for a fixed price in all cells. This kind of unfairness can be countered by a decentralized pricing mechanism. That is, the prices paid per cell should be used to cover the cell operational costs and the proportional core network costs. Besides, the radio resource goods (RRGs) market varies in time, e.g., rush hours. The operator should have the opportunity to assign the RRGs to the users which pay most in order to optimize the operator's profit. Currently,

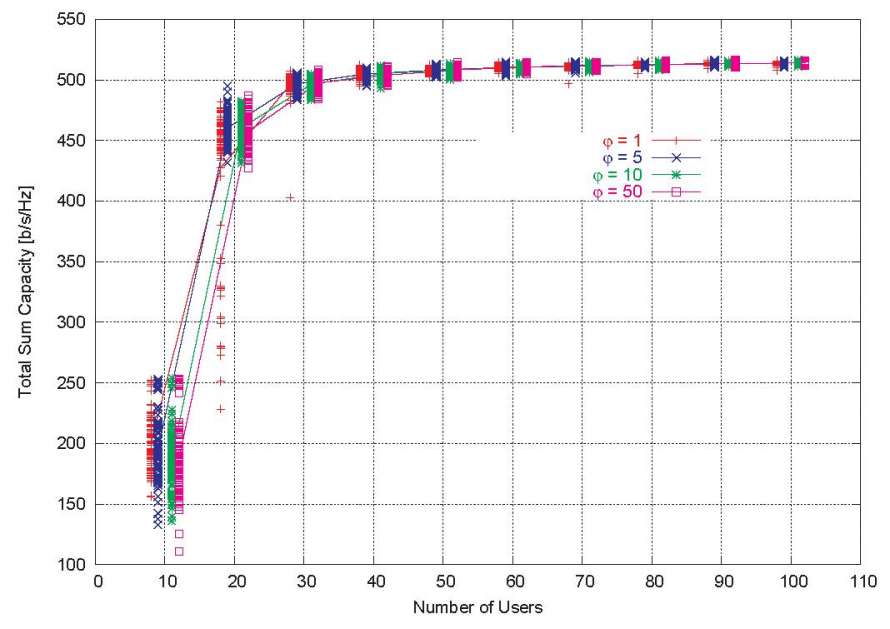

Fig. 5. Total sum capacity as a function of $\varphi$.

resources are allocated in the first-come-first-serve manner, because everybody pays the fixed price. But if someone is willing to pay more for the RRGs, the operator should assign the RRGs to him. The request time should only be used as the second criterion if two users bid the same.

In order to find out the users who are willing to pay the highest prices, negotiations have to take place. As discussed in [9], for each base station an economic manager allocates the RRGs to the user terminals by a discriminatory multi-unit auction sequence. This approach combines the RRG allocation with the economical aspects in the dynamic and distributed market. It can further be envisaged that base stations which are currently not cost-efficient can either be reconfigured to another radio access technology or their RRGs can be rented by another system (even of another operator).

In an auction, the reserve price is the leverage of the operator as the bids are for the users. Therefore, the user terminal needs a bidding strategy which uses the information about load, other bids, and own needs of RRGs for the different services. The goal of the bidding strategy is to win enough RRGs, so that the QoS of different service classes is not injured. The bidding strategy is a medium access protocol and thus is logically located in the second layer of the OSI model. In the following, a bidding strategy is proposed which can be used in the system concept presented in [9]. This bidding strategy combines two machine learning methods, Q-learning and swarm intelligence, in order to learn online and allow non-linear utility functions. For that purpose, a special kind of swarm intelligence which is called particle swarm optimization (PSO) [10] is used.

\section{A. Particle swarm optimization}

PSO [10], [11] is an evolution of the evolutionary algorithm concept. In contrast to genetic algorithms [12] and evolutionary strategies [13], PSO does not imitate the mutations of the genome of a population, but orients itself on the information exchange of a swarm of birds, fishes, or insects. PSO behaves to the classical evolutionary algorithm in the 
same way as evolutionism to the memetics as defined by Dawkins [14]. Therefore, PSO is also called memetics-based algorithm. On the one hand, a gene is the atomic unit of a hereditary character, on the other hand, a mem is the atomic unit of a social construct like an idea or a gesture. The main difference between the classical evolutionary algorithm and PSO is the implemented memory for found values and orientation. Altogether, the implementation effort is less and the manageability is better.

The basic structure resembles the structure of a classical genetic algorithm for which individuals are represented by a vector of real values. The individuals of a PSO are called particles and are randomly initialized. Each particle represents a possible solution of the opimization problem. A particle contains the following information:

- The position of the particle, $\vec{x}$, in the variable space of the optimization problem.

- The evaluation of the fitness function based on the current position in the variable space.

- The velocity vector, $\vec{v}$, which indicates the movement of the particle in the variable space.

- The personal best evaluation of the fitness function during the journey through the variable space.

- The position of the personal best evaluation, $\vec{x}_{p b}$.

- The evaluation of the best fitness function value found from all particles up to this point in time.

- The position of the global best evaluation, $\vec{x}_{g b}$, up to this point in time.

The movement through the variable space proceeds similarly to the one of the classical evolutionary algorithm by evaluating the current position with the fitness function. Each particle compares the current value with its personal best value and overwrites the personal best value if the current value is higher. Hereupon, all personal best values will be compared with the global best value. Again, if there is a global value which is higher than the personal best, the personal best will be overwritten by this value. Afterwards, the velocity in the variable space is calculated by

$$
\vec{v}=\vec{v}+c_{1} a_{1}\left(\vec{x}_{g b}-\vec{x}\right)+c_{2} a_{2}\left(\vec{x}_{p b}-\vec{x}\right),
$$

where $a_{1}$ and $a_{2}$ are uniformly distributed random variables in $[0,1]$ and $c_{1}$ and $c_{2}$ are learning variables which can be adapted by the user. The second addend in (5) tries to turn the particle into the direction to the global best position. The longer the distance between the global best location and the current location of the particle, the higher the incentive to go this direction. The same can be stated for the third addend. That is, the new velocity components will be in direction of the global best and its own best location. The variables $a_{1}$ and $a_{2}$ are used to randomly try another direction, e.g., if one addend dominates the other, because the global best does not mean that the absolut global best has been found. Based on the velocity, $\vec{v}$, the new location is determined by $\vec{x}=\vec{x}+\vec{v}$. The process starts again until a maximum number of runs is reached or the found value is higher than a given threshold.

\section{B. Belief function}

The market is an instationary process, therefore the more time has passed, the smaller the information importance of an observation is. The problem is to estimate the cumulative distribution function by using simple means. One solution is the belief function as proposed in [15]. This method is based on the evidence theory introduced in [16]. The belief function, $b\left(x_{0}\right)$, collects past results of the boolean evaluation of a hypotheses, $f(x)$, of the process and stores them with the process excited value, $x$, and the point of time, $t$, in a limited list. The belief function approximates the cumulative distribution function that the next process result, $f(x)$, will be true by dividing the number of all events, $A\left(x_{0}\right)$, for which $x<x_{0}$ in the list by $A\left(x_{0}\right)$ plus the number of all events, $B\left(x_{0}\right)$, for which $f(x)=$ false and $x \leq x_{0}$ :

$$
b\left(x_{0}\right)=\frac{A\left(x_{0}\right)}{A\left(x_{0}\right)+B\left(x_{0}\right)}
$$

\section{Bidding strategy}

The bidding strategy mainly calculates the bids by applying Q-learning in conjunction with PSO. The learning task is to bid as low as possible for a certain amount of RRGs subject to win enough RRGs to fulfill the QoS. Furthermore, a decision is needed if the RRGs offered by the base station are enough to cover the current demand in this auction period. If this is the case, PSO tries to find an RRG selection for the different QoS classes in order to reduce the number of upcoming data which should necessarily be sent to fulfill the QoS.

The next step of the learning algorithm is to quantize the range from the reserve price to the maximum costs that a bid for a proper QoS class is not allowed to exceed. Based on the belief function, an evidence value, $e(p)$, will be calculated:

$$
e(p)=1-(1-b(p))^{N_{\mathrm{RR} G}},
$$

where it is assumed that all bids are independent. This value is a component of the Q-function in combination with the reward function, $r$, which is the difference of the actual paid price to the maximum costs. Thus, the Q-function, $Q$, is defined as

$$
Q=\sum_{i=1}^{N_{n}} b\left(p_{n}\right)+r, \quad i=1,2, \ldots, N_{n},
$$

where it must be summarized over all required goods. After calculating the $Q$ values, the algorithm chooses the highest value and sends the corresponding bid vector to the base station.

\section{Simulation}

One cell is considered for the simulation of the distributed resource auctioning system. A base station assigns RRGs based on a multi-unit sealed-bid discriminatory auction. The user terminal supports two QoS classes, where QoS 1 has a higher priority than QoS 2 in terms of maximum costs and utility. All user terminals bid with respect to the proposed bidding strategy. In Fig. 6 the absolute reward, $r_{\mathrm{abs}}$, of one user out of 5 users attending the cell is depicted. It shows that 


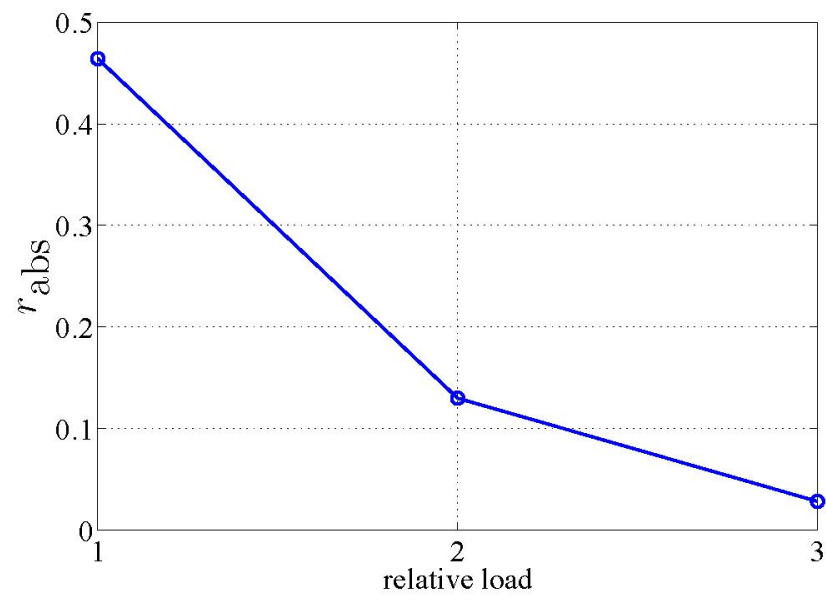

Fig. 6. Bidding strategy behavior indicated by the reward.

the reward will get the smaller, the higher the overall load will be. In turn, based on the increase demand, the bidding strategy possesses the incentive to bid higher. In Fig. 7 the percentage of the RRGs sent divided by the number of required RRGs for each QoS class depending on the number of users attending the cell is shown. It is assumed that each user has the same load. Based on the higher priority and the higher purchase power of QoS 1, the percentage of the sent RRGs is higher than for QoS 2. That is, the bidding strategy tries to prefer the QoS depending on the priority.

\section{CONCLUSION}

We presented bio-inspired approaches for distribution and sharing processes in wireless radio networks. The most important benefits of swarm intelligence at the detection of spectrum holes are time saving and the ability to face changing conditions in the environment. Distributed resource allocation between users in an OFDMA system in uplink in a single cell has shown that the standard deviation of the number of negotiations reduces as the number of the users increases and that a proper tuning of learning and forgetting capabilities has a great impact on the system's negotiation duration. For distributed resource auctioning it can be stated that the reward gets the smaller, the higher the overall load is. In turn, the bidding strategy possesses the incentive to bid a higher value. In addition to that, we have shown that the bidding strategy tries to prefer the quality of service depending on the priority.

\section{ACKNOWLEDGMENT}

This work was performed in project $E^{2} \mathrm{R}$ II which has received research funding from the Community's Sixth Framework program. This paper reflects only the authors' views and the Community is not liable for any use that may be made of the information contained therein. The contributions of colleagues from $E^{2} \mathrm{R}$ II consortium are hereby acknowledged.

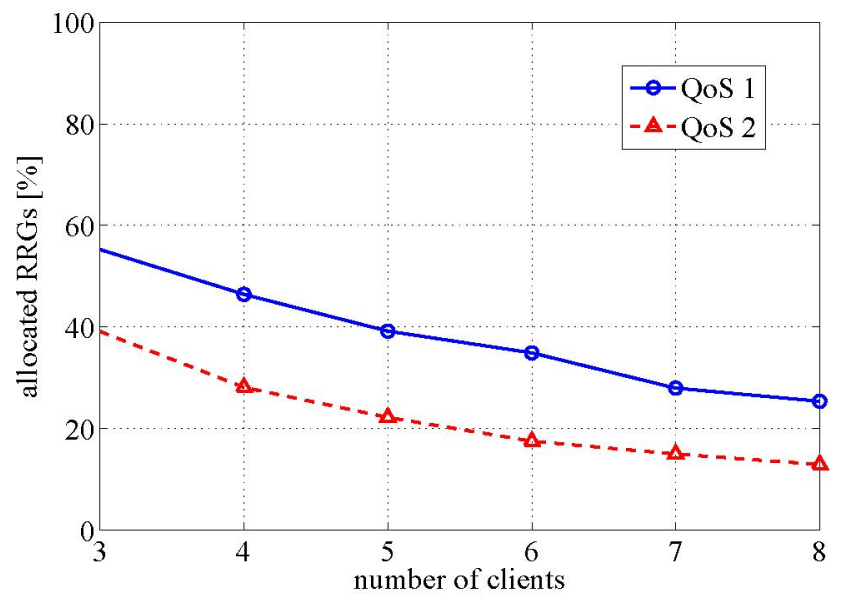

Fig. 7. Percentage of the RRGs sent divided by the number of required RRGs for each QoS class.

\section{REFERENCES}

[1] S. Verdu, "Wireless bandwidth in the making," IEEE Communications Magazine, pp. 53-58, 2000.

[2] Q. Bi, G. I. Zysman, and H. Menkes, "Wireless mobile communications at the start of the 21st century," IEEE Communications Magazine, pp. $110-116,2001$.

[3] M. Roth and S. Wicker, "Termite: Ad-hoc networking with stigmergy," IEEE Global Telecommunications Conference (GLOBECOM O3), 2003.

[4] — " "Termite: Emergent ad-hoc networking," The Second Mediterranean Workshop on Ad-Hoc Networks.

[5] T. Renk, C. Kloeck, and F. K. Jondral, "A cognitive approach to the detection of spectrum holes in wireless networks," IEEE Consumer Communications and Networking Conference (CCNC 07), Las Vegas, USA, 2007.

[6] T. Blackwell and J. Branke, "Multi-swarms, exclusion, and anticonvergence in dynamic environments," IEEE Transactions on Evolutionary Computation, 2006.

[7] V. Cicirello and S. Smith, "Distributed coordination resources via wasplike agents," Workshop on Radical Agent Concepts, 2002.

[8] J. C. Dunat, D. Grandblaise, and C. Bonnet, "Collaborative allocation of orthogonal frequency division multiplex sub carriers using swarm intelligence," Journal of Communications, vol. 1, 2006.

[9] C. Kloeck, H. Jaekel, and F. K. Jondral, "Dynamic and local combined pricing, allocation and billing system with cognitive radios," Proceedings of DySPAN, IEEE, 8-11 Nov. 2005, pp.73 - 81 .

[10] J. Branke, "Evolutionary optimization in dynamic environments," Kluwer Academic Publishers, 2002.

[11] J. Kennedy, R. C. Eberhart, and Y. Shi, "Swarm intelligence," ISBN 1-55860-595-9, Morgan Kaufmann, 2001.

[12] J. H. Holland, "Adaptation in natural and artificial systems," ISBN 0472-08460-7, Univ, of Michigan Pr., 1975.

[13] I. Rechenberg, "Evolutionsstrategie '94," ISBN 3-7728-1642-8, Frommann-Holzboog, 1994.

[14] R. Dawkins, "Evolutionsstrategie '94," ISBN 0-19-857519-X, Oxford Univ, Pr., 1977

[15] S. Gjerstad and J. Dickhaut, "Price Formation in Double Auctions," Lecture Notes in Computer Science, 2001.

[16] G. Shafer, "Price Formation in Double Auctions," ISBN 0-691-08175-1, 0-691-10042-X, Princeton Univ. Pr., 1976. 